study. DNA extraction from peripheral blood samples was performed using the phenol-chloroform method. SNPs were genotyped using the real-time PCR with fluorescent probes. The allele and genotype frequencies were compared using the $x 2$ test. Odds ratios (ORs) and 95\% confidence intervals (95\% Cls) were calculated using the VassarStats online tool.

Results: Utilizing recessive genetic model we found an association between TT genotype of STAT4 rs7574865 (OR = 2.362; 95\%Cl [1.0378 - 5.376], $\mathrm{p}=0.038$ ) and RA. For IL6 rs1800795, it was found that CC genotype had significantly higher frequency among patients with rheumatoid arthritis as compared to that in controls $(\mathrm{OR}=1.52 ; 95 \% \mathrm{Cl}[1.02-2.27], \mathrm{p}=0.0456)$. No associations of IL6R rs2228145 and rs4845618 SNPs with risk of RA were found in the total group of patients vs. controls. It was also shown that IL6 rs 1800795 CC genotype frequency was significantly higher among the patients with RF-negative status ( $p=0.0019)$

Conclusion: Thus, we provide evidence for association of the STAT4 rs7574865 and IL6 rs 1800795 variants with risk of RA in the Belarusian population, some features of interplay being revealed between gene polymorphisms analyzed and RA antibody status. Abovementioned SNPs may contribute to RA genetic susceptibility in the Belarusian population.

Disclosure of Interests: None declared

DOI: 10.1136/annrheumdis-2020-eular.1990

\section{AB0009 ASSOCIATION BETWEEN POLYMORPHISMS OF BANK1 AND MANIFESTATIONS OF SLE}

D. D. González-Castillo ${ }^{1,2}$, R. E. Barbosa-Cobos ${ }^{1}$, G. E. Lugo Zamudio ${ }^{1}$, M. A. Saavedra ${ }^{3}$, R. E. Sánchez-Briones ${ }^{3}$, I. Alemán-Ávila ${ }^{4}$, J. Ramírez Bello ${ }^{4}$. ${ }^{1}$ Hospital Juárez de México, Rheumatology, Ciudad de México, Mexico; ${ }^{2}$ SENESCYT, Guayaquil, Ecuador; ${ }^{3}$ Centro Medico Nacional La Raza, Rheumatology, Ciudad de México, Mexico; ${ }^{4}$ Hospital Juárez de México, Unit of Research, Ciudad de México, Mexico

Background: BANK1 encodes an adapter/scaffold protein primarily expressed in $B$ cells, which is involved in cell signaling and activation. Genome-wide association studies (GWAS) have identified different BANK1 single nucleotide variants (SNVs) associated with SLE primarily in European or Asian-derived populations. Interestingly, we recently have documented an association between this gene and susceptibility to systemic lupus erythematosus (SLE) in Mexican population.

Objectives: To determine whether the BANK1 R61H (rs10516487G/A) and A383T (rs3733197G/A) SNVs are associated with clinical and immunological manifestations in SLE.

Methods: Our study included 123 Mexican women with SLE (SLICC 2012 criteria). Genotyping of the two BANK1 SNVs were obtained by TaqMan probes and real-time PCR. An association study was performed between the alleles and genotypes of BANK1 R61H and A383T with the clinical and immunological manifestations included in the SLE SLICC classification criteria. Hardy-Weinberg equilibrium and an association study was performed using Finetti, a $p$ value $\leq$ 0.05 indicated association.

Results: We identify an average age of $38.5 \pm 12$. Cases and controls remained in Hardy-Weinberg equilibrium. An association with susceptibility to SLE was found between genotypes of the two BANK1 SNVs and joint manifestations (rs1051487G/A; AA + GA vs GG, OR 4.45, $p=0.004$, rs3733197G/A; AA + GA vs GG, OR 2.66, $p=0.032$, respectively), as well as with protection for neurological and renal involvement (rs1051487G/A, OR 0.16, $p=0.02$, rs3733197G/A; OR $0.40, p=0.02$, respectively) (Table $1 \mathrm{a}$ and $b$ ). No association was found with other clinical manifestations.

Conclusion: Our data in the Mexican population show that both BANK1 R61H and A383T SNVs are risk factors for synovitis. On the other hand, these BANK1 $\mathrm{R} 61 \mathrm{H}$ and $\mathrm{A} 383 \mathrm{~T}$ variants are protective factors for neurological and renal damage, respectively.

References:

[1] Ramírez-Bello J, Jiménez-Morales S, Montufar-Robles I, et al. BLK and BANK1 polymorphisms and interactions are associated in Mexican patients with systemic lupus erythematosus. Inflamm Res. 2019;68:705-13

[2] J. De Azevêdo Silva, C. Addobbati, P. Sandrin-Garcia and S. Crovella. Systemic Lupus Erythematosus: Old and New Susceptibility Genes versus Clinical Manifestations. Current Genomics. 2014;15:52-65

[3] Sánchez E, Rasmussen A, Riba L, Acevedo E, Kelly J, Langefeld CD, et al. Impact of Genetic Ancestry and Socio-Demographic Status on the Clinical Expression of Systemic Lupus Erythematosus in Amerindian-European Populations. Arthritis Rheum. 2012; 64(11):3687-3694

[4] Castillejo-López C, Delgado-Vega AM, Wojcik J, et al. Genetic and physical interaction of the B-cell systemic lupus erythematosus-associated genes BANK1 and BLK. Ann Rheum Dis. 2012;71:136-42
[5] Kozyrev SV, Abelson AK, Wojcik J, et al. Functional variants in the B-cell gene BANK1 are associated with systemic lupus erythematosus. Nat Genet. 2008;40:211-6

Disclosure of Interests: None declared

DOI: 10.1136/annrheumdis-2020-eular.5141

\begin{tabular}{|l|l}
\hline AB0010 & TRIPLE DMARD TREATMENT IN EARLY RHEUMATOID \\
ARTHRITIS INCREASE SYNOVIAL ACTIVATED \\
NATURAL KILLERS AND RESTING MAST CELLS BUT \\
DECREASE PLASMA CELLS AND M1 MACROPHAGES
\end{tabular}

M. Y. Hachim ${ }^{1}$, N. M. Elemam ${ }^{1}$, I. Hachim ${ }^{1}$, S. Hannawi ${ }^{2}$, R. Hamoudi ${ }^{1}$, A. A. Maghazachi'. 'Sharjah Institute for Medical Research, College of Medicine, University of Sharjah, Sharjah, United Arab Emirates; ${ }^{2}$ Ministry of Health and Prevention, Department of Rheumatology, Dubai, United Arab Emirates

Background: Rheumatoid arthritis (RA) is a chronic inflammatory joint disease with cartilage and bone damage as well as disability and its optimal therapeutic success depends on understanding the underlying pathophysiology[1]. Since RA is a heterogenous disease, there is an urge to characterize new molecular mechanisms to aid the development of more effective and personalized therapy [2]. Genome-wide transcriptional effects of tDMARD in early RA synovial tissues showed alterations in gene expression of $\mathrm{T}$ cell activation and plasmablast/plasma cell differentiation[3]

Objectives: Using publicly available synovial tissue transcriptomic data to compare the immune cells infiltration at baseline and after 6 months of tDMARD to identify subgroups that might not respond well to tDMARD.

Methods: RNAseq dataset (GSE97165) of synovial biopsies taken from 19 early RA patients at baseline and after 6 months of tDMARD treatment were retrieved and reanalyzed. The raw RNAseq data were used for in silico prediction of the immune cells' infiltration the synovial tissue using CIBERSORT analytical tool to evaluate the pre versus post tDMARD changes in immune population and/or activation status. Then, patients were divided according to the level of alteration in immune cells percentage after the treatment. Differentially expressed genes between the subgroups were defined and gene set enrichment analysis was performed to identify the underlying pathways in each group using BioJupies tools.

Results: 4 immune cells populations showed significant changes after 6 months of tDMARD indicating their role in disease pathophysiology or in response to the therapy. Resting mast cells and activated natural killer (NK) cells were increased in $84 \%$ and $74 \%$ of patients, respectively. On the other hand, M1 macrophages and plasma cells were decreased after treatment in $68 \%$ and $58 \%$ of patients, respectively. GSEA of differentially expressed genes between patients who showed increased activated NK cells in comparison to those who showed decreased or no change in NK cells after treatment identify novel pathways that can explain the heterogeneity in response to treatment specifically genes related to WNT signaling, estrogen metabolism and IL17 signaling.
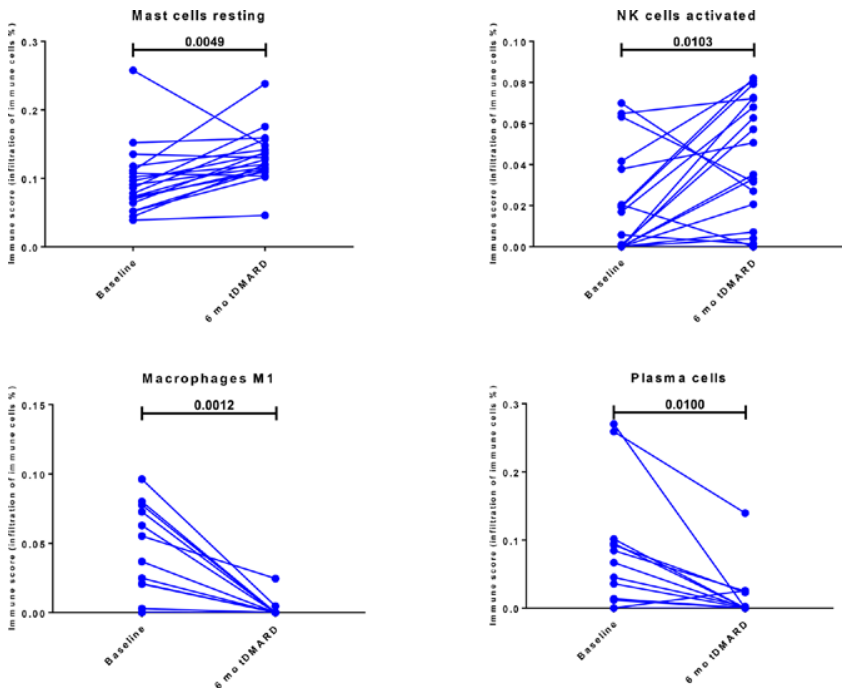

Figure 1. Percentage of infiltrating immune cells in the synovial tissue at baseline and after 6 months of tDMARD therapy in 19 early RA patients using CIBERSORT tool. 